Portland State University

PDXScholar

\title{
Predicted Stresses in Ball-Grid-Array (BGA) and Column-Grid-Array (CGA) Interconnections in a Mirror-Like Package Design
}

\author{
Ephraim Suhir \\ Portland State University \\ Reza Ghaffarian \\ Jet Propulsion Laboratory \\ Johann Nicolics \\ Technische Universität Wien
}

Follow this and additional works at: https://pdxscholar.library.pdx.edu/mengin_fac

Part of the Mechanical Engineering Commons

Let us know how access to this document benefits you.

\section{Citation Details}

Suhir, E., Ghaffarian, R., and Nicolics, J. (2016). Predicted Stresses in Ball-Grid-Array (BGA) and ColumnGrid-Array (CGA) Interconnections in a Mirror-Like Package Design. Journal of Materials Science: Materials in Electronics, Volume 27, Issue 3, pp 2430-2441.

This Article is brought to you for free and open access. It has been accepted for inclusion in Mechanical and Materials Engineering Faculty Publications and Presentations by an authorized administrator of PDXScholar. Please contact us if we can make this document more accessible: pdxscholar@pdx.edu. 


\title{
Predicted stresses in ball-grid-array (BGA) and column-grid- array (CGA) interconnections in a mirror-like package design
}

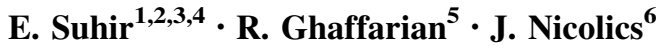

Received: 5 September 2015/Accepted: 5 November 2015/Published online: 25 November 2015

(C) Springer Science+Business Media New York 2015

\begin{abstract}
There is an obvious incentive for using bowfree (temperature change insensitive) assemblies in various areas of engineering, including electron device and electronic packaging fields. The induced stresses in a bow-free assembly could be, however, rather high, considerably higher than in an assembly, whose bow is not restricted. The simplest and trivial case of a bow-free assembly is a tri-component body, in which the inner component is sandwiched between two identical outer components ("mirror" structure), is addressed in our analysis, and a simple and physically meaningful analytical stress model is suggested. It is concluded that if acceptable stresses (below yield stress of the solder material) are achievable, a mirror (bow-free, temperature-change-insensitive) design should be preferred, because it results in an operationally stable performance of the system.
\end{abstract}

E. Suhir

suhire@aol.com

R. Ghaffarian

reza.ghaffarian@jpl.nasa.gov

J. Nicolics

johann.nicolics@tuwien.ac.at

Portland State University, Portland, OR, USA

2 Technische Universität Wien, Vienna, Austria

3 Ariel University, Ariel, Israel

4 ERS Co. LLC, 727 Alvina Ct., Los Altos, CA 94024, USA

5 Jet Propulsion Laboratory, California Institute of Technology, 4800 Oak Grove Dr., Pasadena, CA 91109, USA

6 Department of Applied Electronic Materials, Institute of Sensor and Actuator Systems, Technische Universität Wien, Gusshausstrasse 27-29, 1040 Vienna, Austria

\section{Introduction}

Soldered assemblies are widely used in semiconductor packaging engineering (see, e.g., [1] ). Such assemblies experience, because of the dissimilar materials and change in temperature, thermally induced stresses and deformations that change with the change in temperature. In the majority of cases it is the induced stresses that cause reliability problems in structural elements [2-8], including electron devices and packages [9-17]. There are also situations, when it is the strains, deformations and displacements that are of primary reliability concern. E.g., in many opto-electronics devices and packages elevated stresses might be acceptable (provided that they are still below the yield level), while even small structural displacements (movements) are highly undesirable: if this happens, the functional (optical) performance of the device might be compromised [18]. There is an obvious incentive for designing and using bow-free (temperature change insensitive) assemblies. A bi-material/bi-component assembly cannot be made bow free, because it is statically determinate. The thermally induced forces acting in the crosssections of the components of such an assembly are equal in magnitude, opposite in sign, and create a bending moment that can be equilibrated only by the elastic moment. This inevitably produces non-zero deflections. They can be low, if at least one of the assembly components has a high flexural rigidity, but never zero. To be bow-free, an assembly should be statically indeterminate and contain at least three dissimilar materials (components), so that the resulting bending moment, caused by the induced forces in all the three materials, is zero [20-22].

In the analysis that follows we address the simplest case of a tri-component bi-material assembly, in which the two outer components (packages) are identical. We develop 
simple analytical models for the evaluation of the thermally induced stresses in such a bow-free assembly in application to ball-grid-array (BGA) or column-grid-array (CGA) solder joint interconnections (Fig. 1) employed as suitable attachments between the inner (substrate) and the outer (packages) components.

\section{Analysis}

\subsection{Assumptions}

The following assumptions are used in the analysis.

- A linear elastic approximation can be applied to evaluate the stresses and deformations.

- The actual inhomogeneous BGA or CGA assembly can be replaced with a continuous (homogeneous) bonding layer. This is acceptable, if the gaps between the supports (BGA balls or CGA columns) are small, and the product $k l$ of the parameter $k$ of the interfacial shearing stress and half the assembly length $l$ is significant. Specifically, this could be done, if the ratio $\frac{p}{2 l}$ of the pitch $p$ (distance between the joint centers) to the joint widths $2 l$ is below 5, and the computed product $k l$ is above 2.5 , which is indeed the case in actual BGA and CGA systems.

- Strength-of-material (structural analysis) approach can be employed. As long as such an approach is used, no singular stresses occur at the assembly edges. The predicted stresses evaluated on the basis of the structural analysis approach can be viewed, from the theory-of-elasticity standpoint, as useful design characteristics of the state of stress in the assembly, including its end portions.
- The assembly and its components (constituents) can be treated as thin elongated rectangular plates, experiencing small deflections, and the engineering theory of bending of such plates can be applied to evaluate the states of stress and strain.

- The axial normal stresses in the cross-sections of the assembly components and the interfacial shearing stresses can be determined without taking into account the effect of the peeling stresses. After the interfacial shearing stresses are determined, the peeling stresses can be computed with sufficient accuracy from the calculated shearing stresses.

- The axial normal stresses in the mid-portions of the assembly components can be found on the basis of the conditions of the strain compatibility, without considering the edge effects.

- The interfacial shearing stresses can be evaluated on the basis of the compatibility of the interfacial displacements, using the concept of the interfacial compliance. In accordance with this concept, the longitudinal interfacial displacements can be sought as the sum of (1) the unrestricted (stress free) thermal displacements; (2) displacements caused by the thermally induced forces in the assembly components; these displacements can be found using Hooke's law assuming that, although the cross-sections of the assembly components can rotate, they remain plane (undistorted), when subjected to bending and axial deformations (Kirchhoff-Love hypothesis); (3) displacements due to bending, if any, and additional displacements due to the distortions of the components cross-sections in the proximity of, and at, the interfaces; these "corrections" account for the fact that the interfacial displacements are somewhat larger than the displacements of the inner points of the cross-section and that the sought
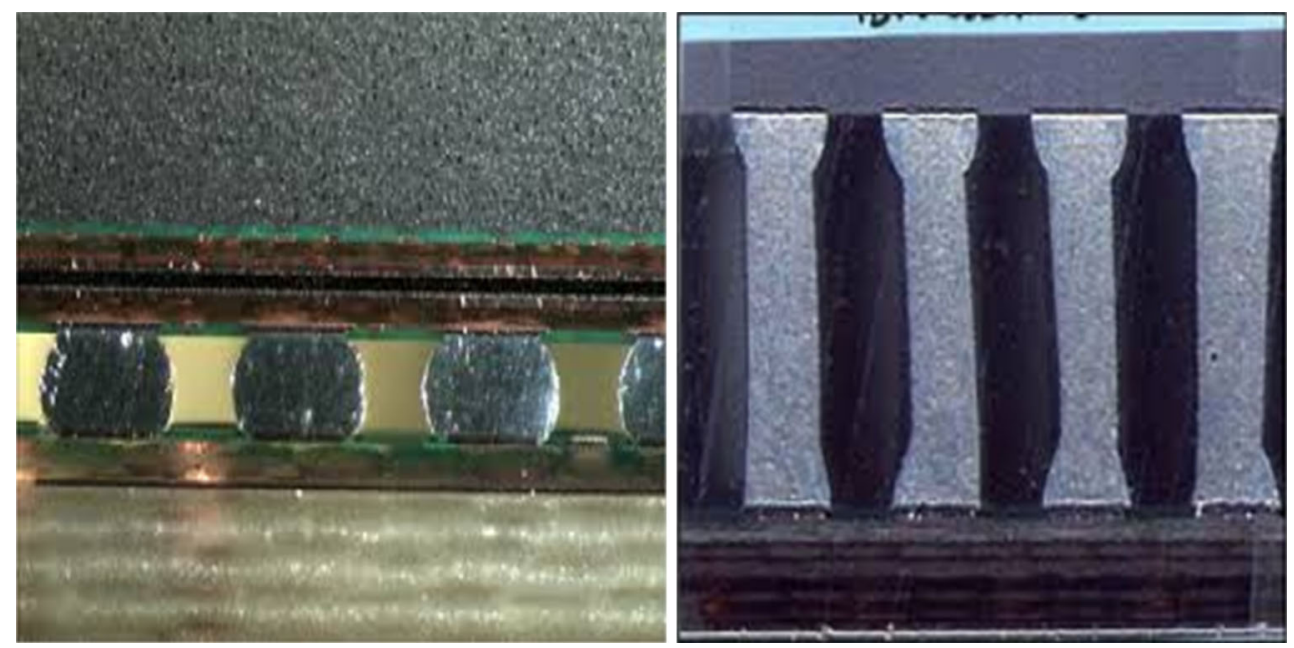

Fig. 1 Key structural elements defining reliability under thermal stress in BGA (on the left) and CGA systems (on the right) are (1) the package (upper component), (2) the PCB (lower component) and (3) the solder joint interconnections 
deviations from planarity are proportional to the level of the shearing stress in the given cross-section and can be represented as a product of the longitudinal interfacial compliance of the component and the shearing stress at the cross-section in question; in other words, it is assumed that the states of stress and strain in the adjacent cross-sections do not affect the distortion in the planarity of the given cross-section; in this approach the longitudinal interfacial compliances are characteristics of the material and the geometry (thickness) of the component and are loading (stress) independent; the interfacial compliances can be evaluated on the basis of the theory-of-elasticity approach (such as, e.g., Ribière solution for a long and narrow strip) for an arbitrary (preferably, simplified, say, constant) load distributed along the component's longitudinal edge(s).

- When the longitudinal interfacial compliance is evaluated and Ribière solution for a long and narrow strip is employed for this purpose, the analysis could be restricted to the longitudinal cross-section of the assembly component, i.e., could be carried out for a long-and-narrow strip of unit width

- The engineering theory of elongated plates (beams) lying on a continuous elastic foundation can be used to evaluate the peeling stresses if necessary.

\subsection{Forces in the assembly mid-portion}

Let a tri-material bow-free assembly be fabricated at an elevated temperature and subsequently cooled down to a low (room, testing, operation) temperature. The conditions

$-\alpha_{1} \Delta t+\lambda_{1} T_{1}=-\alpha_{2} \Delta t+\lambda_{2} T_{2}=-\alpha_{3} \Delta t+\lambda_{3} T_{3}$

of the strain compatibility and the condition

$T_{1}+T_{2}+T_{3}=0$

of equilibrium for the induced forces $T_{i}, \quad i=0,1,2$, should be fulfilled. In these conditions, the components \#\#1 and 3 are the outer ones, the component \#2 is the inner one, $\alpha_{i}, i=1,2,3$, are the coefficients of thermal expansion (CTE) of the materials,

$\lambda_{i}=\frac{1}{E_{i}^{*} h_{i}}, \quad i=1,2,3$,

are the axial compliances of the assembly components, $h_{i}, i=0,1,2$, are their thicknesses,

$E_{i}^{*}=\frac{E_{i}}{1-v_{i}}, \quad i=1,2,3$

are the effective Young's moduli of the materials, $E_{i}, \quad i=$ $1,2,3$, are their actual Young's moduli and $v_{i}, \quad i=$ $0,1,2$, are Poisson's ratios.
Solving the Eqs. (1) and (2) for the forces $T_{i}$, we obtain the following expressions for the forces acting in the components' cross-sections:

$$
\begin{aligned}
& T_{1}=\frac{\left(\alpha_{1}-\alpha_{2}\right) \lambda_{3}+\left(\alpha_{1}-\alpha_{3}\right) \lambda_{2}}{\lambda_{2} \lambda_{1}+\lambda_{1} \lambda_{3}+\lambda_{3} \lambda_{2}} \Delta t, \\
& T_{2}=\frac{\left(\alpha_{2}-\alpha_{1}\right) \lambda_{3}+\left(\alpha_{2}-\alpha_{3}\right) \lambda_{1}}{\lambda_{2} \lambda_{1}+\lambda_{1} \lambda_{3}+\lambda_{3} \lambda_{2}} \Delta t, \\
& T_{3}=\frac{\left(\alpha_{3}-\alpha_{2}\right) \lambda_{1}+\left(\alpha_{3}-\alpha_{1}\right) \lambda_{2}}{\lambda_{2} \lambda_{1}+\lambda_{1} \lambda_{3}+\lambda_{3} \lambda_{2}} \Delta t .
\end{aligned}
$$

As has been shown in the previous report that addressed the case of a single substrate, the induced force in a particular component is next-to-zero, if this component's axial compliance is significantly greater than the compliance of the two other components. This is indeed the case for a bimaterial assembly with a compliant bond provided by the BGA or CGA system. In the assembly addressed in this report the packages and the substrate have comparable axial compliances, so that all the three thermally induced forces have to be considered. On the other hand, the estimated axial compliances of the BGA or CGA systems that provide attachment between these major "players"-the two packages and the substrate-are significant compared to the compliances of the major assembly components and the thermal mismatch of these systems with the major assembly components need not be accounted for. It is only their interfacial compliance that should be considered, when evaluating the induced interfacial stresses.

When the components \#1 and \#3 are identical, the formulas (5) yield:

$T_{1}=\frac{\alpha_{1}-\alpha_{2}}{\lambda_{1}+2 \lambda_{2}} \Delta t, T_{2}=2 \frac{\alpha_{2}-\alpha_{1}}{\lambda_{1}+2 \lambda_{2}} \Delta t$

\subsection{Zero bow condition}

No assembly bow could possibly occur, if the bending moment produced by these forces with respect to any longitudinal axis is zero. If one requires, e.g., that the bending moment is zero with respect to the mid-plane of the inner component, then the condition

$T_{1} \frac{h_{1}+h_{2}}{2}-T_{3} \frac{h_{2}+h_{3}}{2}=0$.

should be fulfilled. This relationship, considering the first and the third formulas in (5), results in the following condition of zero bow:

$$
\begin{aligned}
& E_{2}^{*} E_{1}^{*} h_{2} h_{1}\left(h_{2}+h_{1}\right)\left(\alpha_{1}-\alpha_{2}\right) \\
& \quad+E_{1}^{*} E_{3}^{*} h_{1} h_{3}\left(h_{1}+2 h_{2}+h_{3}\right)\left(\alpha_{1}-\alpha_{3}\right) \\
& \quad-E_{2}^{*} E_{3}^{*} h_{2} h_{3}\left(h_{2}+h_{3}\right)\left(\alpha_{3}-\alpha_{2}\right)=0
\end{aligned}
$$

The condition (8) is always fulfilled, of course, in the case of identical outer components, like in the addressed 
"mirror" assembly. Let us consider, however, two other, less obvious, special cases for this condition.

1. $h_{2}=0$ (the inner component does not exist). In this case the condition (8) yields: $\alpha_{1}=\alpha_{3}$. Hence, in such a bimaterial assembly the condition of zero bow requires that the two remaining components have the same CTE. The same result could be obtained when either the component $\# 1$ or the component \#3 does not exist, i.e., when $h_{1}=0$ or $h_{3}=0$.

2. $\alpha_{1}=\alpha_{3}$ (the two outer components have the same CTE). In this case the condition (8) yields:

$\frac{h_{2}\left(h_{2}+h_{3}\right)}{h_{1}\left(h_{2}+h_{1}\right)}=\frac{E_{1}^{*}}{E_{3}^{*}}$.

Thus, the difference in the effective Young's moduli should be compensated by the adequate thicknesses of the components and the elastic constants of the materials. If the inner component is very thin compared to the outer components, then the formula (9) yields: $\sqrt{\frac{h_{3}}{h_{1}}}=\frac{E_{1}^{*}}{E_{3}^{*}}$. In the opposite extreme case, when the inner component is significantly thicker than the outer components, $\frac{h_{3}}{h_{1}}=\frac{E_{1}^{*}}{E_{3}^{*}}$.

If one intends, e.g., to choose the appropriate thickness of the bonding material with characteristics

$E_{2}=741.24 \mathrm{~kg} / \mathrm{mm}^{2}=7264 \mathrm{GPa} ; v_{2}=0.42$,

$E_{2}^{*}=900 \mathrm{~kg} / \mathrm{mm}^{2}=8820 \mathrm{Gpa} ; \alpha_{2}=60 \times 10^{-6} 1 /{ }^{\circ} \mathrm{C}$,

to attach a $h_{1}=0.5 \mathrm{~mm}=0.0005 \mathrm{~m}$ thick elongated $\mathrm{Si}$ plate with characteristics

$E_{1}=11,309 \mathrm{~kg} / \mathrm{mm}^{2}=110,828 \mathrm{Gpa}, v_{1}=0.24$,

$E_{1}^{*}=12,000 \mathrm{~kg} / \mathrm{mm}^{2}=117.600 \mathrm{Gpa}, \alpha_{1}=2.5 \times 10^{-6} 1 /{ }^{\circ} \mathrm{C}$,

to an elongated silica glass substrate

$E_{3}=6912 \mathrm{~kg} / \mathrm{mm}^{2}=67,737 \mathrm{GPa}, v_{3}=0.20$,

$E_{3}^{*}=7200 \mathrm{~kg} / \mathrm{mm}^{2}=70,560 \mathrm{GPa}, \alpha_{3}=0.5 \times 10^{-6} 1 /{ }^{\circ} \mathrm{C}$,

of the given thickness, then the condition (8) suggests that the bonding layer should be $h_{2}=0.2408 \mathrm{~mm}=$ $0.0002408 \mathrm{~m}$ thick for a $h_{3}=0.3322 \mathrm{~mm}=0.0003322 \mathrm{~m}$ thick glass substrate. The calculated data indicate, particularly, that there is no need to make the bonding layer unreasonably thick to create a large enough thermal force (stress) in it in order to achieve a bow-free effect.

\subsection{Parameter of the interfacial shearing stress}

The longitudinal interfacial displacements can be sought, in an approximate analysis based on the theory of interfacial compliances and in accordance with the taken assumptions, as follows:

$$
\begin{aligned}
& u_{12}(x)=-\alpha_{1} \Delta t x+\lambda_{1} \int_{0}^{x} T_{1}(x) d x-\kappa_{1} \tau_{12}(x), \\
& u_{21}(x)=-\alpha_{2} \Delta t x+\lambda_{2} \int_{0}^{x} T_{2}(x) d x+\kappa_{2} \tau_{12}(x), \\
& u_{23}(x)=-\alpha_{2} \Delta t x+\lambda_{2} \int_{0}^{x} T_{2}(x) d x+\kappa_{2} \tau_{23}(x), \\
& u_{32}(x)=-\alpha_{3} \Delta t x+\lambda_{3} \int_{0}^{x} T_{3}(x) d x-\kappa_{3} \tau_{23}(x) .
\end{aligned}
$$

Here $u_{12}(x)$ are the longitudinal displacements of the outer component \#1 at its interface with the inner component \#2, $u_{21}(x)$ are the displacements of the inner component \#2 at its interface with the component \#1, $u_{23}(x)$ are the displacements of the inner component \#2 at its interface with the component \#3, $u_{32}(x)$ are the displacements of the outer component \#3 at its interface with the inner component \#2, $T_{i}(x), i=1,2,3$ are the distributed forces acting in the assembly components cross-sections,

$\tau_{12}(x)=T_{1}^{\prime}(x), \tau_{23}(x)=T_{3}^{\prime}(x)$,

are the shearing stresses at the interfaces between the components \#\#1 and 2, and at the components \#\#2 and 3, respectively,

$\kappa_{1}=\frac{h_{1}}{3 G_{1}}, \kappa_{2}=\frac{h_{2}}{6 G_{2}}, \kappa_{3}=\frac{h_{3}}{3 G_{3}}$

are the longitudinal interfacial compliances of the assembly components, and

$G_{i}=\frac{E_{i}}{2\left(1+v_{i}\right)}, \quad i=1,2,3$

are shear moduli of the component materials. The formulas (11) follow from the obvious relationships

$T_{1}(x)=\int_{-l}^{x} \tau_{12}(\xi) d \xi, T_{3}(x)=\int_{-l}^{x} \tau_{23}(\xi) d \xi$

where $l$ is half the assembly length. The first and the third formulas in (12) are obtained based on the Ribière solution in the theory-of-elasticity for a long-and-narrow strip loaded in the antisymmetric fashion along one of its long sides. The second formula in (12) was obtained for a longand-narrow strip loaded in an antisymmetric fashion along both the long sides of the strip. The origin of the coordinate $x$ is in the mid-cross-section of the assembly.

The displacement compatibility conditions can be written for the assembly components as follows: 
$u_{12}(x)=u_{21}(x)+\kappa_{12} \tau_{12}(x), u_{32}(x)=u_{23}(x)+\kappa_{23} \tau_{23}(x)$,

where

$\kappa_{12}=\frac{h_{12}}{G_{12}}, \kappa_{23}=\frac{h_{23}}{G_{23}}$

are the longitudinal interfacial compliances of the bonding layers between the components \#1 and \#2, and the components \#2 and \#3, respectively, $h_{12}$ and $h_{23}$ are thicknesses of these layers (actually, the heights/standoffs of the BGA and CGA interconnections), and $G_{12}$ and $G_{23}$ are shear moduli of the materials.

Considering that the forces (5) are in equilibrium, and therefore

$T_{2}(x)=-\left[T_{1}(x)+T_{3}(x)\right]$,

we obtain the following basic equations for the sought interfacial stresses:

$$
\begin{gathered}
\kappa_{12}^{*} \tau_{12}(x)-\left(\lambda_{1}+\lambda_{2}\right) \int_{0}^{x} T_{1}(\xi) d \xi \\
-\lambda_{2} \int_{0}^{x} T_{3}(\xi) d \xi=\left(\alpha_{2}-\alpha_{1}\right) \Delta t x, \\
\kappa_{23}^{*} \tau_{23}(x)-\lambda_{2} \int_{0}^{x} T_{1}(\xi) d \xi-\left(\lambda_{2}+\lambda_{3}\right) \\
\int_{0}^{x} T_{3}(\xi) d \xi=\left(\alpha_{2}-\alpha_{3}\right) \Delta t x,
\end{gathered}
$$

where

$\kappa_{12}^{*}=\kappa_{1}+\kappa_{2}+\kappa_{12}, \kappa_{23}^{*}=\kappa_{2}+\kappa_{3}+\kappa_{23}$

are the total compliances of the interfaces between the components \#1 and \#2, and \#2 and \#3. In the "mirror" design in question, when the two outer components and the two compliant attachments (strain buffering layers) are identical, a single equation

$\kappa_{12}^{*} \tau_{12}(x)-\left(\lambda_{1}+2 \lambda_{2}\right) \int_{0}^{x} T_{1}(\xi) d \xi=\left(\alpha_{2}-\alpha_{1}\right) \Delta t x$.

can be considered, instead of the two Eqs. (18) and (19).

By differentiating the Eqs. (18) we have:

$\kappa_{12}^{*} \tau_{12}^{\prime}(x)-\left(\lambda_{1}+\lambda_{2}\right) T_{1}(x)-\lambda_{2} T_{3}(x)=\left(\alpha_{2}-\alpha_{1}\right) \Delta t$,

$\kappa_{23}^{*} \tau_{23}^{\prime}(x)-\lambda_{2} T_{1}(x)-\left(\lambda_{2}+\lambda_{3}\right) T_{3}(x)=\left(\alpha_{2}-\alpha_{3}\right) \Delta t$.

The next differentiation yields: $\kappa_{12}^{*} \tau_{12}^{\prime \prime}(x)-\left(\lambda_{1}+\lambda_{2}\right) \tau_{12}(x)-\lambda_{2} \tau_{23}(x)=0$,

$\kappa_{23}^{*} \tau_{23}^{\prime \prime}(x)-\lambda_{2} \tau_{12}(x)-\left(\lambda_{2}+\lambda_{3}\right) \tau_{23}(x)=0$.

The interfacial shearing stresses must be anti-symmetric with respect to the mid-cross-section of the assembly, and the solutions to these equations could be sought in the form:

$\tau_{12}(x)=C_{1} \sinh k x, \tau_{23}(x)=C_{2} \sinh k x$,

where $k$ is just far unknown parameter of the interfacial shearing stresses. Introducing the solutions (23) into the Eqs. (22) we obtain the following homogeneous algebraic equations for the constants $C_{1}$ and $C_{2}$ :

$$
\begin{aligned}
{\left[\kappa_{12}^{*} k^{2}-\left(\lambda_{1}+\lambda_{2}\right)\right] C_{1}-\lambda_{2} C_{2} } & =0, \\
-\lambda_{2} C_{1}+\left[\kappa_{23}^{*} k^{2}-\left(\lambda_{2}+\lambda_{3}\right)\right] C_{2} & =0 .
\end{aligned}
$$

Equating the determinant of these equations to zero, we conclude that the parameter $k$ of the interfacial shearing stress can be determined from the following bi-quadratic equation:

$k^{4}-\left(k_{12}^{2}+k_{23}^{2}\right) k^{2}+(1-\delta) k_{12}^{2} k_{23}^{2}=0$,

where

$$
\begin{aligned}
k_{12} & =\sqrt{\frac{\lambda_{1}+\lambda_{2}}{\kappa_{12}^{*}}}, k_{23}=\sqrt{\frac{\lambda_{2}+\lambda_{3}}{\kappa_{23}^{*}}}, \delta \\
& =\frac{\lambda_{2}^{2}}{\left(\lambda_{1}+\lambda_{2}\right)\left(\lambda_{2}+\lambda_{3}\right)}
\end{aligned}
$$

are, respectively, the parameter of the interfacial shearing stress for a bi-material assembly comprised of the components \#1 and \#2, the parameter of the interfacial shearing stress for an assembly comprised of the components \#2 and \#3, and the parameter of the relative axial compliance of the inner component.

The bi-quadratic Eq. (25) has the following solution:

$k=\sqrt{\frac{k_{12}^{2}+k_{23}^{2}}{2}\left[1 \pm \sqrt{1-(1-\delta)\left(\frac{2 k_{12} k_{23}}{k_{12}^{2}+k_{23}^{2}}\right)^{2}}\right]}$.

In order to establish which sign should be accepted in front of the inner root, let us consider a special case when the axial compliance of the inner component is significantly greater than the compliances of the two outer components. Then the parameter $\delta$ is close to one, and only the sign "+" leads to the finite value of the parameter $k$ When all the three components are identical, i.e., when $k_{12}=k_{23}=k_{0}$ and $\delta=\frac{1}{4}$, then the solution (27) yields: $k=$ $\sqrt{\frac{3}{2}} k_{0}$. Thus, an assembly with three identical components is characterized by the parameter $k$ of the interfacial 
shearing stress that is by the factor of $k=\sqrt{\frac{3}{2}}=1.2247$ greater than in the case of a bi-component assembly.

\subsection{The case of identical outer components}

We seek the coordinate $x$ dependent thermally induced force in the inner component in the form

$T(x)=T\left(1-\frac{\cosh k x}{\cosh k l}\right)$.

where $T$ is the force acting in the mid-cross-sections of this component and expressed by the formulas (6), and the parameter $k$ of the interfacial shearing stress is expressed by the formula (27). The expression (28) satisfies the zero boundary condition at the assembly ends, and, for large enough $k l$ products, becomes independent of the longitudinal position of the particular cross-section, as long as it is in the mid-portion of the assembly.

The formulas (14) suggest that the corresponding interfacial shearing stress is next-to-zero in the mid-portion of the assembly and can be found as

$\tau(x)=T^{\prime}(x)=-k T \frac{\sinh k x}{\cosh k l}$

at its peripheral portions. The maximum shearing stresses take place at the end cross-sections $x= \pm l$ :

$\tau_{\max }=-k T \tanh k l$.

For long enough assemblies with stiff interfaces $(k l \geq 2.5)$ this formula yields:

$\tau_{1, \max }=-k T_{1}, \tau_{3, \max }=-k T_{3}$.

In a mirror type assembly these stresses are equal, of course.

\subsection{Peeling stresses: basic equations}

Although the assembly is bow-free, the peeling stresses could nonetheless be appreciative, since the outer components could deflect with respect to the inner component. Let us assume that the peeling stresses are proportional to these deflections:

$p_{12}(x)=K_{12} w_{1}(x), p_{23}(x)=K_{23} w_{2}(x)$

The interfacial spring constants $K_{12}$ and $K_{23}$ in the through-thickness direction can be assessed by the approximate formulas

$$
\begin{aligned}
K_{12} & =\frac{1}{\frac{1-v_{12}}{E_{12}} h_{12}+\frac{1-v_{1}}{3 E_{1}} h_{1}+\frac{1-v_{2}}{3 E_{2}} h_{2}}, K_{23} \\
& =\frac{1}{\frac{1-v_{23}}{E_{23}} h_{23}+\frac{1-v_{2}}{3 E_{2}} h_{2}+\frac{1-v_{3}}{3 E_{3}} h_{3}}
\end{aligned}
$$

Treating the outer components of the assembly as elongated rectangular plates, the following equations of equilibrium (bending) for these components can be applied:

$D_{1} w_{1}^{\prime \prime}(x)=\frac{h_{1}}{2} T_{1}(x)-\int_{0}^{x} \int_{0}^{x} p_{12}(\xi) d \xi d \xi$,

$D_{3} w_{3}^{\prime \prime}(x)=\frac{h_{3}}{2} T_{3}(x)-\int_{0}^{x} \int_{0}^{x} p_{23}(\xi) d \xi d \xi$

where

$D_{1}=\frac{E_{1} h_{1}^{3}}{12\left(1-v_{1}^{2}\right)}, D_{3}=\frac{E_{3} h_{3}^{3}}{12\left(1-v_{3}^{2}\right)}$

are the flexural rigidities of the outer components. The left parts of the Eq. (33) are the elastic bending moments caused by bending. The first terms in the right parts of these equations are the bending moments due to the thermally induced forces $T_{1}(x)$ and $T_{3}(x)$. The second terms are the bending moment caused by the peeling stresses.

Excluding the deflection functions $w_{1}(x)$ and $w_{3}(x)$ from the Eqs. (32) and (34) we obtain the following basic equations for the peeling stress functions $p_{12}(x)$ and $p_{23}(x)$ :

$p_{12}^{\prime \prime}(x)+4 \beta_{12}^{4} \int_{0}^{x} \int_{0}^{x} p_{12}(\xi) d \xi d \xi=4 \beta_{12}^{4} \frac{h_{1}}{2} T_{1}(x)$,

$p_{23}^{\prime \prime}(x)+4 \beta_{23}^{4} \int_{0}^{x} \int_{0}^{x} p_{23}(\xi) d \xi d \xi=4 \beta_{23}^{4} \frac{h_{2}}{2} T_{3}(x)$.

Here $\beta_{12}=\sqrt[4]{\frac{K_{12}}{4 D_{1}}}$ and $\beta_{23}=\sqrt[4]{\frac{K_{23}}{4 D_{3}}}$ are the parameters of the peeling stresses. Since the two Eq. (36) are similar, the further analysis is carried out in application to the first equation in (36). In the final results the index " 12 " could be simply replaced with the index " 23 ".

Differentiating the first equation in (36) twice we have:

$p_{12}^{I V}(x)+4 \beta_{12}^{4} p_{12}(x)=4 \beta_{12}^{4} p_{012} \frac{\cosh k x}{\cosh k l}$,

where the notation

$p_{012}=\frac{h_{1}}{2} \frac{\alpha_{2}-\alpha_{1}}{\kappa_{1}+\kappa_{2}} \Delta t$.

is used. This Eq. (37) has the form of an equation that is used in the theory of beams supported by elastic foundations. The difference is, however, that the Eq. (37) is obtained for the peeling stress, not for the deflection function. 


\subsection{Peeleing stresses: solutions to the basic equations}

The solution to the Eq. (37) can be sought in the form:

$p_{12}(x)=C_{0} V_{0}(\beta x)+C_{2} V_{2}(\beta x)+\frac{\eta_{12}^{4}}{1+\eta_{12}^{4}} p_{012} \frac{\cosh k x}{\cosh k l}$,

where

$\eta_{12}=\frac{\beta_{12} \sqrt{2}}{k}$

is the ratio of the parameters of the peeling and the shearing interfacial stresses, and the functions $V_{i}(\beta x), i=$ $0,1,2,3$, are expressed as

$$
\begin{aligned}
V_{0}(\beta x) & =\cosh \beta x \cos \beta x, \\
V_{2}(\beta x) & =\cosh \beta x \cos \beta x, \\
V_{1,3}(\beta x) & =\frac{1}{\sqrt{2}}(\cosh \beta x \sin \beta x \pm \sinh \beta x \cos \beta x)
\end{aligned}
$$

The constants $C_{0}$ and $C_{1}$ of integration in the solution (39) can be found as

$$
\begin{aligned}
& C_{0}=\frac{2 \eta_{12}}{1+\eta_{12}^{4}} p_{012} e^{-u}\left[\sqrt{2} \cos u+\eta_{12}(\sin u-\cos u)\right], \\
& C_{2}=\frac{2 \eta_{12}}{1+\eta_{12}^{4}} p_{012} e^{-u}\left[\sqrt{2} \sin u-\eta_{12}(\sin u+\cos u)\right],
\end{aligned}
$$

and the solution (39) results in the following expression for the peeling stress:

$$
\begin{aligned}
& p_{12}(x)=\frac{\eta_{12}}{1+\eta_{12}^{4}} p_{01}\left[\eta_{12}^{3} e^{-k(l-x)}+4 e^{-\beta_{12}(l-x)}\right. \\
& \left.\quad\left(\left(\sqrt{2}-\eta_{12}\right) \cos \beta_{12}(l-x)+\eta_{12} \sin \beta_{12}(l-x)\right)\right]
\end{aligned}
$$

Similarly, we obtain:

$$
\begin{aligned}
& p_{23}(x)=\frac{\eta_{23}}{1+\eta_{23}^{4}} p_{023}\left[\eta_{23}^{3} e^{-k(l-x)}+4 e^{-\beta_{23}(l-x)}\right. \\
& \left.\quad\left(\left(\sqrt{2}-\eta_{23}\right) \cos \beta_{23}(l-x)+\eta_{23} \sin \beta_{23}(l-x)\right)\right]
\end{aligned}
$$

At the assembly ends $x=1$

$$
\begin{aligned}
p_{12}(l) & =\frac{\eta_{12}}{1+\eta_{12}^{4}} p_{012}\left(\eta_{12}^{3}+4\left(\sqrt{2}-\eta_{12}\right)\right), p_{23}(l) \\
& =\frac{\eta_{23}}{1+\eta_{23}^{4}} p_{02}\left(\eta_{23}^{3}+4\left(\sqrt{2}-\eta_{23}\right)\right)
\end{aligned}
$$

from the boundary conditions $p_{12}^{\prime \prime}(l)=0, p_{12}^{\prime \prime \prime}(l)=0$. These conditions follow from the first of the assumed relationships (32), since no concentrated bending moments, nor lateral forces act at the free end of the assembly, and therefore the second and the third derivatives of the deflection function should be zero. These boundary conditions are equivalent to the conditions

$$
\int_{0}^{l} \int_{0}^{x} p_{1}(\xi) d \xi d \xi=0, \int_{0}^{l} p_{1}(x) d x=0
$$

of self-equilibrium of the peeling stress. The notation

$u=\beta_{12} l=l \sqrt[4]{\frac{K_{12}}{4 D_{1}}}$

is used in the solutions (42).

In the practically important case of an elongated assembly the formulas (42) can be simplified:
When the parameters $\eta_{12}$ and $\eta_{23}$ are significant, $p_{12}(l)=p_{012}$ and $p_{23}(l)=p_{023}$. This result explains the physical meaning of the $p_{012}$ and $p_{023}$ values: these are the peeling stresses at the ends of a long assembly with very stiff through-thickness interfaces.

After the peeling stresses are determined, the deflections can be found as

$w_{1}(x)=\frac{p_{12}(x)}{K_{12}}, w_{3}(x)=\frac{p_{23}(x)}{K_{23}}$

The maximum deflections are

$w_{1}(l)=\frac{p_{12}(l)}{K_{12}}, w_{3}(l)=\frac{p_{23}(l)}{K_{23}}$.

\section{Numerical example}

The calculation procedure below could be followed, when a structure with two identical outer components is employed, and compliant bonds are provided by a BGA or a CGA system. 


\subsection{Input data}

\begin{tabular}{llll}
\hline Structural element & Package & PCB & $\begin{array}{l}\text { Solder } \\
3-4 \% \mathrm{Ag} 0.5-1 \% \mathrm{Cu} \\
12 \text { and } 23\end{array}$ \\
Element number & & & 5510.0 \\
Young'smodulus, $E, \mathrm{~kg} / \mathrm{mm}^{2}(\mathrm{GPa})$ & 1 and 3 & 2 & $(53.998 \mathrm{GPa})$ \\
& 8775.5 & 2321.4 & 0.35 \\
Poisson's ratio, $v$ & $(86.0 \mathrm{GPa})$ & $(22.750 \mathrm{GPa})$ & $\mathrm{x}$ \\
$\mathrm{CTE} \alpha, 1 /{ }^{\circ} \mathrm{C}$ & 0.25 & 0.40 & $0.60 / \mathrm{BGA}$ \\
Thickness, $h, \mathrm{~mm}(\mathrm{~m})$ & $6.5 \times 10^{-6}$ & $15.0 \times 10^{-6}$ & $2.20 / \mathrm{CGA}$ \\
& 2.0 & 1.5 & 2040.7 \\
Shear modulus, $G, \mathrm{~kg} / \mathrm{mm}^{2}(\mathrm{GPa})$ & $(0.0020 \mathrm{~m})$ & $(0.0015 \mathrm{~m})$ & $(20.0 \mathrm{GPa})$ \\
& 3367.3 & 892.7 & $\mathrm{x}$ \\
Axial compliance, $\lambda, \mathrm{mm} / \mathrm{kg}(\mathrm{m} / \mathrm{N})$ & $(33.0 \mathrm{GPa})$ & $(8.748 \mathrm{GPa})$ & $20.1028 \times 10^{-5}$ \\
Interfacial compliance, $\kappa, \mathrm{mm} / \mathrm{kg}\left(\mathrm{m}^{3} / \mathrm{N}\right)$ & $3.9884 \times 10^{-5}$ & $\left(0.1970 \times 10^{-5}\right)$ & $29.4017 \times 10^{-5} / \mathrm{BGA}$ \\
& $\left(390.86 \times 10^{-5}\right)$ & $56.0099 \times 10^{-5}$ & $107.8061 \times 10^{-5} / \mathrm{CGA}$ \\
Flexural rigidity, $D, \mathrm{~kg} / \mathrm{mm}^{3}(\mathrm{Nm})$ & $19.7982 \times 10^{-5}$ & $\left(5.7152 \times 10^{-14}\right)$ & -
\end{tabular}

Estimated yield stress of the solder material in shear $\tau_{Y}=1.85 \mathrm{~kg} / \mathrm{mm}^{2}=18.130 \mathrm{MPa}$

Soldering temperature $230{ }^{\circ} \mathrm{C}$; Assumed change in temperature $\Delta t=200{ }^{\circ} \mathrm{C}$;

Half package (assembly) length $l=15 \mathrm{~mm}=0.05 \mathrm{~m}$

\subsection{Calculated data}

Axial compliances of the assembly components (\#1 and \#3 are identical outer components; \#2 is the inner component):

$$
\begin{aligned}
\lambda_{1,3} & =\frac{1-v_{1}}{E_{1} h_{1}}=\frac{1-0.25}{8775.5 \times 2.0} \\
& =4.2733 \times 10^{-5} \mathrm{~mm} / \mathrm{kg}=4.1878 \times 10^{-7} \mathrm{~m} / \mathrm{N} ; \\
\lambda_{2} & =\frac{1-v_{2}}{E_{2} h_{2}}=\frac{1-0.40}{2321.4 \times 1.5}=17.2310 \times 10^{-5} \mathrm{~mm} / \mathrm{kg} \\
& =16.8862 \times 10^{-7} \mathrm{~m} / \mathrm{N} ;
\end{aligned}
$$

Interfacial compliances of the assembly components:

$$
\begin{aligned}
\kappa_{1,3} & =\frac{h_{1}}{3 G_{1}}=\frac{2.0}{3 \times 3367.3}=19.7983 \times 10^{-5} \mathrm{~mm}^{3} / \mathrm{kg} \\
& =1,940,233.4 \mathrm{~m}^{3} / \mathrm{N}, \\
\kappa_{2} & =\frac{h_{2}}{6 G_{2}}=\frac{1.5}{6 \times 892.7}=28.0049 \times 10^{-5} \mathrm{~mm}^{3} / \mathrm{kg} \\
& =2,744,480.2 \mathrm{~m}^{3} / \mathrm{N}
\end{aligned}
$$

Interfacial compliances of the solder systems (on each side of the inner component):

$$
\begin{aligned}
\kappa_{12} & =\frac{h_{12}}{G_{12}}=\frac{0.6}{2040.7}=29.4017 \times 10^{-5} \mathrm{~mm}^{3} / \mathrm{kg} \\
& =2,881,366.6 \mathrm{~m}^{3} / \mathrm{N}
\end{aligned}
$$

in the case of BGA, and

$$
\begin{aligned}
\kappa_{12} & =\frac{h_{12}}{G_{12}}=\frac{2.2}{2040.7}=107.8061 \times 10^{-5} \mathrm{~mm}^{3} / \mathrm{kg} \\
& =10,564,997.8 \mathrm{~m}^{3} / \mathrm{N}
\end{aligned}
$$

in the case of for CGA. Clearly, the solder systems provide considerable additional interfacial compliance to the designs. The interfacial compliance of the CGA system is significantly, by the factor of 3.7 , larger than that of the BGA.

The total compliance of the interface between the outer (package) and the inner (PCB) components is

$$
\begin{aligned}
\kappa_{12}^{*} & =\kappa_{12}+\kappa_{1}+\kappa_{2}=77.2049 \times 10^{-5} \mathrm{~mm}^{3} / \mathrm{kg} \\
& =7,566,080.2 \mathrm{~m}^{3} / \mathrm{N}
\end{aligned}
$$

in the case of BGA system, and

$$
\begin{aligned}
\kappa_{12}^{*} & =\kappa_{12}+\kappa_{1}+\kappa_{2}=155.6093 \times 10^{-5} \mathrm{~mm}^{3} / \mathrm{kg} \\
& =15,249,711.4 \mathrm{~m}^{3} / \mathrm{N}
\end{aligned}
$$

in the case of CGA system. The total interfacial compliance of the system with CGA interfaces is about twice as large as the compliance of the assembly with the BGA system.

The "local" parameter of the interfacial shearing stress (i.e., the parameter of the interfacial shearing stress for a bi-component assembly that consists of the components \#1 and \#2 only) is 


$$
\begin{aligned}
k_{12} & =\sqrt{\frac{\lambda_{1}+\lambda_{2}}{\kappa_{12}^{*}}}=\sqrt{\frac{21.5043 \times 10^{-5}}{77.2049 \times 10^{-5}}}=0.5278 \mathrm{~mm}^{-1} \\
& =527.8 \mathrm{~m}^{-1}
\end{aligned}
$$

in the case of the BGA system and

$$
\begin{aligned}
k_{12} & =\sqrt{\frac{\lambda_{1}+\lambda_{2}}{\kappa_{12}^{*}}}=\sqrt{\frac{21.5043 \times 10^{-5}}{155.6093 \times 10^{-5}}}=0.3717 \mathrm{~mm}^{-1} \\
& =371.7 \mathrm{~m}^{-1}
\end{aligned}
$$

in the case of the CGA system.

The parameter that considers the relative axial compliance of the inner component is

$\delta=\left(1+\frac{\lambda_{1}}{\lambda_{2}}\right)^{-2}=(1+0.1984)^{-2}=0.6420$

If all the assembly components would have the same axial compliance, this parameter would be 0.25 . If the inner component were significantly more compliant than the two outer components, this parameter would be equal to 1.0.

The "global" parameter of the interfacial shearing stress (for the entire assembly) is

$$
\begin{aligned}
k & =k_{12} \sqrt{1+\sqrt{\delta}}=0.5278 \sqrt{1+0.8013}=0.7084 \mathrm{~mm}^{-1} \\
& =708.4 \mathrm{~m}^{-1}
\end{aligned}
$$

in the case of BGA attachment, and

$$
\begin{aligned}
k & =k_{12} \sqrt{1+\sqrt{\delta}}=0.3717 \sqrt{1+0.8013}=0.4989 \mathrm{~mm}^{-1} \\
& =498.9 \mathrm{~m}^{-1}
\end{aligned}
$$

in the case of CGA interconnections. It is by the factor of $\sqrt{1+\sqrt{\delta}}=\sqrt{1+0.8013}=1.342$ higher than the "local" parameter of the interfacial shearing stress is. The product $k l$ is large in both cases: it is $k l=10.6260$ in the case of BGA interconnections and is $k l=7.4835$ in the CGA case. Hence, the actual assembly could be treated in the analysis as an infinitely long one.

The induced forces are

$$
\begin{aligned}
T_{1} & =T_{3}=\frac{\alpha_{1}-\alpha_{2}}{\lambda_{1}+2 \lambda_{2}} \Delta t=-\frac{8.5 \times 10^{-6}}{387.3530 \times 10^{-6}} \times 200 \\
& =-4.3888 \mathrm{~kg} / \mathrm{mm}=-0.0430 \mathrm{~N} / \mathrm{m} \\
T_{2} & =2 \frac{\alpha_{2}-\alpha_{1}}{\lambda_{1}+2 \lambda_{2}} \Delta t=2 \frac{8.5 \times 10^{-6}}{387.3530 \times 10^{-6}} \times 200 \\
& =8.7775 \mathrm{~kg} / \mathrm{mm}=0.0860 \mathrm{~N} / \mathrm{m}
\end{aligned}
$$

Thus, the two outer components are in compression and the inner component is in tension. Clearly, the induced force in the inner component is twice as high as the forces in the outer components.

The normal stresses

$$
\begin{aligned}
& \sigma_{1,3}=\frac{T_{1}}{h_{1}}=-\frac{4.3888}{2.0}=-2.1944 \mathrm{~kg} / \mathrm{mm}^{2}=-21,505 \mathrm{MPa} \\
& \sigma_{2}=\frac{T_{2}}{h_{2}}=\frac{8.7775}{1.5}=5.8517 \mathrm{~kg} / \mathrm{mm}^{2}=57,346 \mathrm{MPa}
\end{aligned}
$$

in the midportions of the components are not affected by the compliant attachments.

The maximum interfacial shearing stress is

$$
\begin{aligned}
\tau_{\max } & =-k T_{1}=0.7084 \times 4.3888=3.1090 \mathrm{~kg} / \mathrm{mm}^{2} \\
& =30,468 \mathrm{MPa}
\end{aligned}
$$

in the case of BGA, and

$$
\begin{aligned}
\tau_{\max } & =k T_{1}=0.4989 \times 4.3888=2.1896 \mathrm{~kg} / \mathrm{mm}^{2} \\
& =21,458 \mathrm{MPa}
\end{aligned}
$$

in the case of CGA. The level of these stresses is comparable to the level of the normal stresses in the components' cross-sections. Thus, the application of the CGA resulted in about $29.6 \%$ relief in the maximum interfacial shearing stress as compared to the BGA based design. In the case of a single substrate these stresses were considerably lower: $\tau_{\max }=1.9483 \mathrm{~kg} / \mathrm{mm}^{2}=19,093 \mathrm{MPa}$ and $\tau_{\max }=1.4747 \mathrm{~kg} / \mathrm{mm}^{2}=14,452 \mathrm{MPa}$, respectively. The application of the "mirror" design results in about $59.6 \%$ increase in the maximum interfacial shearing stress in the case of BGA and in about $48.5 \%$ increase-in the case of a CGA system.

With the assumed yield in shear of $\tau_{Y}=1.85 \mathrm{~kg} / \mathrm{mm}^{2}=$ $18,130 \mathrm{MPa}$ of the solder material no low-cycle fatigue conditions are expected to occur only in the single-substrate design using CGA system. It is noteworthy in this connection that lead-free solders are characterized by considerably higher yield stresses than tin-lead solders. This circumstance might be viewed as an important merit of lead-free solders.

The calculations that follow have been carried out in connection with the evaluation of the peeling stress. Throughthickness stiffness (spring constant) of the solder system is

$$
\begin{aligned}
K_{12} & =\frac{1}{\frac{1-v_{12}}{E_{12}} h_{12}+\frac{1-v_{1}}{3 E_{1}} h_{1}+\frac{1-v_{2}}{3 E_{2}} h_{2}} \\
& =\frac{1}{\frac{1-0.35}{5510.0} 0.6+\frac{1-0.25}{3 \times 8775.5} 2.0+\frac{1-0.40}{3 \times 2321.4} 1.5} \\
& =3891.2111 \mathrm{~kg} / \mathrm{mm}^{3}=3.8134 \times 10^{13} \mathrm{~N} / \mathrm{m}^{3}
\end{aligned}
$$

in the case of BGA, and

$$
\begin{aligned}
K_{12} & =\frac{1}{\frac{1-v_{12}}{E_{12}} h_{12}+\frac{1-v_{1}}{3 E_{1}} h_{1}+\frac{1-v_{2}}{3 E_{2}} h_{2}} \\
& =\frac{1}{\frac{1-0.35}{5510.0} 2.2+\frac{1-0.25}{3 \times 8775.5} 2.0+\frac{1-0.40}{3 \times 2321.4} 1.5} \\
& =2243.4747 \mathrm{~kg} / \mathrm{mm}^{3}=2.1986 \times 10^{13} \mathrm{~N} / \mathrm{m}^{3}
\end{aligned}
$$

in the case of CGA. 
Parameter of the peeling stress (the inner component does not flex and its flexural rigidity could be assumed therefore infinitely large in our calculations) is

$$
\begin{aligned}
\beta_{12} & =\sqrt[4]{\frac{K_{12}}{4 D_{1}}}=\sqrt[4]{\frac{3891.2111}{4 \times 6240.3556}}=0.6283 \mathrm{~mm}^{-1} \\
& =628.3 \mathrm{~m}^{-1}
\end{aligned}
$$

in the case of BGA, and

$$
\begin{aligned}
\beta_{12} & =\sqrt[4]{\frac{K_{12}}{4 D_{1}}}=\sqrt[4]{\frac{2243.4747}{4 \times 6240.3556}}=0.5475 \mathrm{~mm}^{-1} \\
& =547.5 \mathrm{~m}^{-1}
\end{aligned}
$$

in the case of CGA. The factors $\beta_{12} l$ are significant in both BGA and CGA cases, so that the assembly can be treated, when evaluating the peeling stresses, as an infinitely long one.

Peeling stress at the end of an assembly with an infinitely large through-thickness spring constant is

$$
\begin{aligned}
p_{012} & =\frac{\Delta \alpha \Delta t}{\kappa_{12}^{*}} \frac{h_{1}}{2}=\frac{0.00170}{77.2049 \times 10^{-5}} \times \frac{2.0}{2} \\
& =2.2019 \mathrm{~kg} / \mathrm{mm}^{2}=21,579 \mathrm{MPa}
\end{aligned}
$$

in the case of a BGA system, and

$$
\begin{aligned}
p_{012} & =\frac{\Delta \alpha \Delta t}{\kappa_{12}^{*}} \frac{h_{1}}{2}=\frac{0.00170}{155.6093 \times 10^{-5}} \times \frac{2.0}{2} \\
& =1.0925 \mathrm{~kg} / \mathrm{mm}^{2}=10,706 \mathrm{MPa}
\end{aligned}
$$

in the case of CGA. The difference should be attributed to the greater longitudinal interfacial compliance of the CGA system.

The ratio $\eta_{12}$ that characterizes the relative level of the peeling stress parameter with respect to the shearing stress parameter is

$\eta_{12}=\frac{\beta_{12} \sqrt{2}}{\mathrm{k}}=\frac{0.6283 \times 1.4142}{0.7084}=1.2543$

in the case of BGA, and

$\eta_{12}=\frac{\beta_{12} \sqrt{2}}{\mathrm{k}}=\frac{0.5475 \times 1.4142}{0.4989}=1.5520$

in the case of CGA. Note that for a single substrate design the above ratios were $\eta_{12}=1.4850$ and $\eta_{12}=1.8368$, respectively. These data indicate that the role of the peeling stresses, as compared to the interfacial shearing stresses, is greater for the single substrate design than for the "mirror" design.

The predicted peeling stress is

$$
\begin{aligned}
p_{12}(l) & =\frac{\eta_{12}}{1+\eta_{12}^{4}} p_{012}\left(\eta_{12}^{3}+4\left(\sqrt{2}-\eta_{12}\right)\right) \\
& =\frac{1.2543}{3.4752}(2.2019)(1.9733+0.6396) \\
& =2.0765 \mathrm{~kg} / \mathrm{mm}^{2}=20,350 \mathrm{MPa}
\end{aligned}
$$

at the end of the BGA system and is

$$
\begin{aligned}
p_{12}(l) & =\frac{\eta_{12}}{1+\eta_{12}^{4}} p_{012}\left(\eta_{12}^{3}+4\left(\sqrt{2}-\eta_{12}\right)\right) \\
& =\frac{1.5520}{6.8018}(0.9258)(3.7383-0.5512) \\
& =0.6732 \mathrm{~kg} / \mathrm{mm}^{2}=6597 \mathrm{MPa}
\end{aligned}
$$

at the end of the CGA system. These stresses are as high as $94 \%$ of the peeling stress in an assembly with infinitely high through-thickness spring constant in the case of a BGA and as about $73 \%$ in the case of a CGA system.

The obtained data indicate that the peeling stress at the end of the CGA system is only about $32.4 \%$ of the peeling stress at the end of the BGA system. Application of the CBA system in the mirror design had, in the carried out example, a much greater effect on the peeling stress (by about a factor of three) than on the shearing stress (by about $29.6 \%$ ). In the case of a single substrate the peeling stresses were significantly lower than in the mirror design: $p(l)=0.6810 \mathrm{~kg} / \mathrm{mm}^{2}=6674 \mathrm{MPa}$ in the case of the BGA (by the factor of about three) and $p(l)=$ $0.3444 \mathrm{~kg} / \mathrm{mm}^{2}=3375 \mathrm{MPa}$ in the case of the CGA (by the factor of about two).

The maximum bows of the peripheral components (with respect to the inner component) are

$$
\begin{aligned}
w_{\max } & =\frac{p_{12}(l)}{K_{12}}=\frac{1.9884}{1590.3131}=1.2503 \mu \mathrm{m} \\
& =1,2503 \times 10^{-6} \mathrm{~m}
\end{aligned}
$$

in the case of a BGA and

$$
\begin{aligned}
w_{\max } & =\frac{p_{12}(l)}{K_{12}}=\frac{0.8238}{1222.2623}=0.6740 \mu \mathrm{m} \\
& =0.6740 \times 10^{-6} \mathrm{~m}
\end{aligned}
$$

in the case of a CGA. They are certainly significantly lower than the predicted bow of $65.9 \mu \mathrm{m}$ of a single substrate assembly. The obtained results are summarized in the following table: 


\begin{tabular}{|c|c|c|c|c|}
\hline \multirow{2}{*}{$\begin{array}{l}\text { Design } \\
\text { Solder }\end{array}$} & \multicolumn{2}{|c|}{ Single substrate } & \multicolumn{2}{|l|}{ Mirror } \\
\hline & BGA & CGA & BGA & CGA \\
\hline Maximum shearing stress, $\tau_{\max }, \mathrm{kg} / \mathrm{mm}^{2}(\mathrm{MPa})$ & $\begin{array}{l}1.9483 \\
(19.093)\end{array}$ & $\begin{array}{l}1.4747 \\
(14.452)\end{array}$ & $\begin{array}{l}3.1090 \\
(30.468)\end{array}$ & $\begin{array}{l}2.1896 \\
(21.458)\end{array}$ \\
\hline Maximum peeling stress, $p_{\max }, \mathrm{kg} / \mathrm{mm}^{2}(\mathrm{MPa})$ & $\begin{array}{l}0.6810 \\
(6.674)\end{array}$ & $\begin{array}{l}0.3444 \\
(3.375)\end{array}$ & $\begin{array}{l}2.0765 \\
(20,350)\end{array}$ & $\begin{array}{l}0.6732 \\
(6.596)\end{array}$ \\
\hline Maximum bow, $w_{\max } \mu \mathrm{m}(\mathrm{m})$ & $\begin{array}{l}65.9 \\
(0.00006\end{array}$ & & $\begin{array}{l}1.250 \\
(0.00000125)\end{array}$ & $\begin{array}{l}0.674 \\
(0.000000674)\end{array}$ \\
\hline
\end{tabular}

Based on these data, it could be concluded that only the single substrate design, and preferably the one with CGA interconnections, will perform within the elastic region. It should be noted that the difference, if positive, between the predicted elastic stress and the "available" yield stress determines not only the very fact of the existence of the inelastic strains, but also the length of the zone occupied by such strains [23] and the magnitude of the inelastic strains and two identical packages) assembly, have been developed.

- The methodology is presented in the form of a numerical example carried out for typical lead-free BGA and CGA systems. The calculated data are compared with the results of the analysis that addressed the case of a simple substrate, when bowing is expected and permitted. The obtained data are as follows:

\begin{tabular}{|c|c|c|c|c|}
\hline \multirow{2}{*}{$\begin{array}{l}\text { Design } \\
\text { Solder }\end{array}$} & \multicolumn{2}{|c|}{ Single substrate } & \multicolumn{2}{|l|}{ Mirror design } \\
\hline & BGA & CGA & BGA & CGA \\
\hline Maximum shearing stress, $\tau_{\max }, \mathrm{kg} / \mathrm{mm}^{2}(\mathrm{MPa})$ & $\begin{array}{l}1.9483 \\
(19.093)\end{array}$ & $\begin{array}{l}1.4747 \\
(14.452)\end{array}$ & $\begin{array}{l}3.1090 \\
(30.468)\end{array}$ & $\begin{array}{l}2.1896 \\
(21.458)\end{array}$ \\
\hline Maximum peeling stress, $p_{\max }, \mathrm{kg} / \mathrm{mm}^{2}(\mathrm{MPa})$ & $\begin{array}{l}0.6810 \\
(6.674)\end{array}$ & $\begin{array}{l}0.3444 \\
(3.375)\end{array}$ & $\begin{array}{l}2.0765 \\
(20.350)\end{array}$ & $\begin{array}{l}0.6732 \\
(6.597)\end{array}$ \\
\hline Maximum bow, $w_{\max }, \mu \mathrm{m}(\mathrm{m})$ & $\begin{array}{l}65.9 \\
(0.00006\end{array}$ & & $\begin{array}{l}1.250 \\
(0.00000125)\end{array}$ & $\begin{array}{l}0.674 \\
(0.000000674)\end{array}$ \\
\hline
\end{tabular}

at the particular location, and this, in turn, affects the lifetime of the interconnection.

\section{Conclusions}

The following major conclusions can be drawn from the carried out analysis:

- The induced stresses in a bow-free assembly could be rather high, and the elevated stresses could generate undesirable inelastic stresses and strains in low yield stress materials, if any. If this happens, not only the bow-free condition will be compromised, but, more importantly, low cycle fatigue conditions will occur.

- Simple, easy-to-use and physically meaningful analytical predictive stress models, when ball-grid-array (BGA) or column-grid-array (CGA) solder joint interconnections are employed as suitable attachments between the inner (substrate) and the outer (packages) components of such a tri-component (two packages and one substrate) bi-material (materials of the substrate
- If, e.g., the yield stress in shear is $1.85 \mathrm{~kg} /$ $\mathrm{mm}^{2}=18.130 \mathrm{MPa}$ for the solder material considered, then only the single substrate design with the CGA system will operate within the elastic range.

- It should be emphasized, however, that if low enough stresses (below yield stress of the solder material) are achievable, a mirror (bow-free) design is preferable: it will be both inelastic-strain-free and temperaturechange-insensitive, thereby increasing dramatically the fatigue lifetime of the vulnerable material.

- Based on the calculated data, it could be concluded that only the single substrate design, and preferably the one with CGA interconnections, will perform within the elastic region.

- The difference, if positive, between the predicted elastic stress and the "available" yield stress determines not only the very fact of the existence of the inelastic strains, but also the length of the zone occupied by such strains and the magnitude of the inelastic strains at the particular location, and this, in turn, affects the lifetime of the interconnection. 


\section{References}

1. R.R. Tummala (ed.), Fundamentals of Microsystems Packaging (McGraw-Hill, New York, 2001)

2. S.P. Timoshenko, Analysis of bi-metal thermostats. J. Opt. Soc. Am. 11 (1925)

3. B.J. Aleck, Thermal stresses in a rectangular plate clamped along an edge. ASME J. Appl. Mech. 16 (1949)

4. S. Strinivas, Analysis of Bonded Joints. NASA Technical Note D-7855 (1975)

5. K. Roll, Analysis of stress and strain distribution in thin films and substrates. J. Appl. Phys. 47(7) (1976)

6. F.-V. Chang, Thermal contact stresses of bi-metal strip thermostat. Appl. Math. Mech. 4(3) (1983)

7. E. Suhir, Stresses in bi-metal thermostats. ASME J. Appl. Mech. 53(3) (1986)

8. E. Suhir, Interfacial stresses in bi-metal thermostats. ASME J. Appl. Mech. 56(3) (1989)

9. G.A. Lang et al., Thermal fatigue in silicon power devices. IEEE Trans. Electron. Dev. 17 (1970)

10. R. Zeyfang, Stresses and strains in a plate bonded to a substrate: semiconductor devices. Solid State Electron. 14 (1971)

11. J.H. Lau (ed.), Thermal Stress and Strain in Microelectronics Packaging (Van-Nostrand Reinhold, New York, 1993)

12. E. Suhir, Analysis of interfacial thermal stresses in a tri-material assembly. J. Appl. Phys. 89(7) (2001)

13. J.H. Lau, S.W. Lee (eds.), Chip Scale Packages: Design, Materials, Processes, Reliability, and Applications (McGraw-Hill, New York, 1999)
14. E. Suhir, A. Shakouri, Assembly bonded at the ends: could thinner and longer legs result in a lower thermal stress in a thermoelectric module (TEM) design? ASME J. Appl. Mech. 79(6) (2012)

15. E. Suhir, Thermal stress failures in electronics and photonics: physics, modeling, prevention. J. Therm. Stress. (2013)

16. E. Suhir, D. Shangguan, L. Bechou, Predicted thermal stresses in a tri-material assembly with application to silicon-based photovoltaic module. ASME J. Appl. Mech. 80 (2013)

17. E. Suhir, Thermal stress in through-silicon-vias: theory-of-elasticity approach. Microelectron. Reliab. 54 (2014)

18. E. Suhir, S. Kang, J. Nicolics, C. Gu, A. Bensoussan, L. Bechou, Analytical stress model for the evaluation of thermal stresses in a cylindrical tri-material body with application to optical fibers. J. Electr. Control Eng. 3(5) (2013)

19. E. Suhir, J. Weld, Electronic package with reduced bending stress. US Patent \#5,627,407 (1997)

20. E. Suhir, Arrangement for reducing bending stress in an electronic package. US Patent \#6,810,241 (2001)

21. E. Suhir, Device and method of controlling the bowing of a soldered or adhesively bonded assembly. US Patent \#6,239,382 (2001)

22. E. Suhir, Bow free adhesively bonded assemblies: predicted stresses. Electrotech. Informationtech. 120(6) (2003)

23. E. Suhir, L. Bechou, B. Levrier, D. Calvez, Assessment of the size of the inelastic zone in a BGA assembly. 2013 IEEE Aerospace Conference, Big Sky, Montana, March 2013 